\title{
Level-by-level Artificial Viscosity and Visualization for MHD Simulation with Adaptive Mesh Refinement
}

\author{
Tomoharu Hatori ${ }^{\mathrm{a}}$, Atsushi M. Ito ${ }^{\mathrm{a}, \mathrm{b}}$, Masanori Nunami ${ }^{\mathrm{a}, \mathrm{b}}$, Hideyuki Usui ${ }^{\mathrm{c}}$, \\ and Hideaki Miura ${ }^{a, b}$ \\ ${ }^{a}$ SOKENDAI(The Graduate University for Advanced Studies), Toki, Japan \\ ${ }^{b}$ National Institute for Fusion Science, Toki, Japan \\ ${ }^{c}$ The Graduate School of System Informatics, Kobe University, Kobe, Japan
}

\section{Abstract}

We propose a numerical method to determine the artificial viscosity in magnetohydrodynamics (MHD) simulations with adaptive mesh refinement (AMR) method, where the artificial viscosity is adaptively changed due to the resolution level of the AMR hierarchy. Although the suitable value of the artificial viscosity depends on the governing equations and the model of target problem, it can be determined by von Neumann stability analysis. By means of the new method, "level-by-level artificial viscosity method," MHD simulations of Rayleigh-Taylor instability (RTI) are carried out with the AMR method. The validity of the level-by-level artificial viscosity method is confirmed by the comparison of the linear growth rates of RTI between the AMR simulations and the simple simulations with uniform grid and uniform artificial viscosity whose resolution is the same as that in the highest level of the AMR simulation. Moreover, in the nonlinear phase of RTI, the secondary instability is clearly observed where the hierarchical data structure of AMR calculation is visualized as high resolution region floats up like terraced fields. In the applications of the method to general fluid simulations, the growth 
of small structures can be sufficiently reproduced, while the divergence of numerical solutions can be suppressed.

Keywords: adaptive mesh refinement, magnetohydrodynamics, Rayleigh-Taylor instability, artificial viscosity

\section{Introduction}

The simplest way to perform numerical simulations for field equations with high accuracy is to employ high resolution grids for fields. The problem of the high resolution grids for fields is that computational resources for the simulations increase with the number of grid points. Adaptive mesh refinement (AMR) method is a powerful tool for allocating high resolution grids only to the region having complicated field structure within limited computational resources. The region having simple field structure is calculated with low-resolution grids in simulations with the AMR method. Thus, the AMR method can save the computational resource for the numerical simulation of fields.

The AMR method had been proposed by Berger and Oliger [1], and has been applied into numerical simulations for various subjects of physics, such as hydrodynamics [2-9], magnetohydrodynamics [10-19], cosmology[20-26], geophysics/astrophysics [10-12, 19, 25, 27-46], atmospheric modeling[47],

ocean modeling[48, 49], FDTD(electromagnetic wave)[50], and fusion science[51, $52]$.

However, the AMR method also has disadvantages. One of the disadvantages of the AMR method is the difficulty of implementation of algorithms to manage the adaptive grid. Memory management for creation and deletion of 
the adaptive grid is complicated in most cases. Especially important, careful treatment is needed on the boundary of gris of different size. This point also makes it more difficult to parallelize simulation codes with the AMR method than those with uniform grid. To overcome these difficulties, packages to utilize the AMR method have been developed. For example, BoxLib[53], Cactus[54, 55], Chombo[56], PARAMESH[57, 58], and Uintah[59] are publicly available, and used in various applications [19, 26, 35, 39, 45, 60, 61]. Users of those packages can develop simulation codes with the AMR method more easily than develop simulation codes from scratch.

We too are developing a new AMR package called "Package of Amr for a Variety of Numerical Experiments (PAVANE)." PAVANE can easily integrate conventional simulation codes with the AMR grid system. It autonomously manage the hierarchical data structure and parallelize the codes. This makes it possible to free users from worry about the complexity of the AMR algorithm. Almost the same effort is needed to write a code with PAVANE as to write a simple serial code.

In this paper, we focus on the magnetohydrodynamics (MHD) simulation of Rayleigh-Taylor instability (RTI) in plasma. In the previous works, the secondary instability of RTI had been observed by using MHD simulation with high-resolution and uniform grid $[62,63]$. In the present work, we apply PAVANE to the MHD simulation code, and we have encountered an issue of treatment of artificial viscosity on the fluid simulation with AMR. In fluid simulations, artificial viscosity play an important role. It guarantee numerical stability by smoothing fine structures in the field. To perform high-accuracy simulations, artificial viscosity should be sufficiently small depending on the 
resolution. We propose a new technique, "level-by-level artificial viscosity," to resolve that issue of treatment of artificial viscosity in the simulation with the AMR method.

The rest of this paper is organized as follows. In Section 2, the system of extended MHD equations and simulation conditions are introduced. In Section 3, level-by-level artificial viscosity method is introduced. In Section 4, results and discussion are presented. In Section 5, visualization of AMR simulation is presented. Section 6 summarize this paper.

\section{MHD Equations, Initial condition, and Utilizing PAVANE}

\subsection{MHD equations}

In this paper, we use MHD equations as follows.

$$
\begin{aligned}
& \frac{\partial \rho}{\partial t}=-\nabla \cdot[\rho \boldsymbol{v}]+\mu\left(\frac{\partial^{4}}{\partial x^{4}}+\frac{\partial^{4}}{\partial y^{4}}\right) \rho \\
& \frac{\partial}{\partial t}(\rho \boldsymbol{v})=-\nabla \cdot\left[\rho \boldsymbol{v} \boldsymbol{v}+\mathbf{I}\left(p+\frac{B^{2}}{2}\right)-\boldsymbol{B B}\right]+\rho \boldsymbol{g}+\mu\left(\frac{\partial^{4}}{\partial x^{4}}+\frac{\partial^{4}}{\partial y^{4}}\right)(\rho \boldsymbol{v})
\end{aligned}
$$

$$
\frac{\partial E_{t o t}}{\partial t}=-\nabla \cdot\left[\boldsymbol{v}\left(E_{t o t}+p+\frac{B^{2}}{2}\right)-\boldsymbol{B}(\boldsymbol{v} \cdot \boldsymbol{B})\right]+\rho \boldsymbol{g} \cdot \boldsymbol{v}+\mu\left(\frac{\partial^{4}}{\partial x^{4}}+\frac{\partial^{4}}{\partial y^{4}}\right) E_{t o t}
$$

$$
\frac{\partial \boldsymbol{B}}{\partial t}=-\nabla \cdot[\boldsymbol{v} \boldsymbol{B}-\boldsymbol{B} \boldsymbol{v}]+\mu\left(\frac{\partial^{4}}{\partial x^{4}}+\frac{\partial^{4}}{\partial y^{4}}\right) \boldsymbol{B}
$$

$E_{t o t}=\left(\frac{\rho \boldsymbol{v}^{2}}{2}+\frac{p}{\gamma-1}+\frac{B^{2}}{2}\right)$

$\nabla \cdot \boldsymbol{B}=0$

where $\rho$ is the density, $\boldsymbol{v}$ is the velocity, $p$ is the pressure, $\boldsymbol{B}$ is the magnetic field, $\gamma(=5 / 3)$ is the specific heat ratio, and $\boldsymbol{g}$ is the external accelera- 
tion. The last terms of the right hand side of eq(1)-(4) is artificial viscosity term composed of fourth order differential whose magnitude is determined by artificial viscosity $\mu$. The equation (6) is Gauss's law for magnetism. Spatial differential is numerically replaced by fourth-order central-finite difference method. Time evolution of the eq.(1)-(4) is numerically solved by fourth-order Runge-Kutta-Gill method. Since all physical quantities are dimensionless quantities, a unit of time scale is the Alfven time $\tau_{A}$.

\subsection{Initial Condition of $M H D$}

To bring the RTI on the MHD, the system must have the density gradient initially. In addition, the pressure gradient is also required for force equilibrium against the external acceleration $\boldsymbol{g}$ where $\boldsymbol{g}=(0,-0.01,0)$ in the present paper. Therefore, in this simulation, the system have initially the pressure gradient and density gradient at the center of the simulation box in the y-direction (see fig. 1). The initial profiles of the pressure and density are given by

$$
\begin{aligned}
& \rho(y)=\frac{\rho_{1}+\rho_{2}}{2}+\frac{\rho_{2}-\rho_{1}}{2} \tanh \left(\frac{2 y}{d}\right) \\
& p(y)=\beta\left[\frac{p_{1}+p_{2}}{2}+\frac{p_{2}-p_{1}}{2} \tanh \left(\frac{2 y}{d}\right)\right]
\end{aligned}
$$

where $\rho_{1}$ and $\rho_{2}$ are the lower density and the upper density, $p_{1}$ and $p_{2}$ are the lower pressure and the upper pressure, respectively, $d$ is the width of the gradient, and $\beta$ is the ratio of the pressure to the magnetic pressure. The magnetic field $\boldsymbol{B}$, which is perpendicular to the density and pressure gradients, is given to satisfy the force equilibrium in y-direction,

$$
\frac{\partial}{\partial y}\left[p(y)+\frac{B_{z}^{2}(y)}{2}\right]=-\rho(y) g .
$$




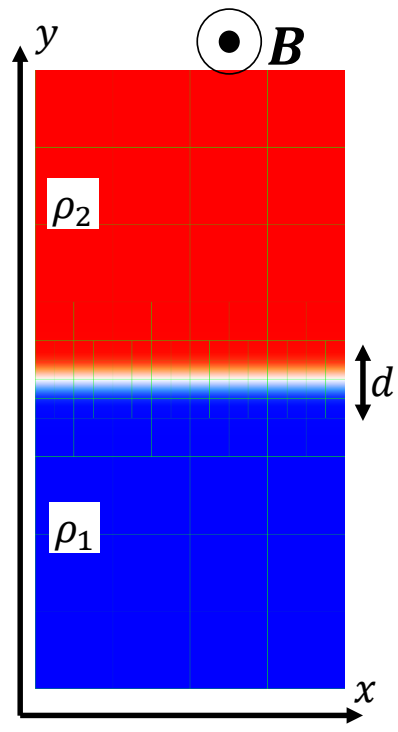

Figure 1: Initial condition for RTI simulations. The density and pressure shear is at the center in the y-direction. The width of the shear is d. The magnetic field is in the z-direction.

The parameters are set as $\rho_{1}=1.0, \rho_{2}=1.1, p_{1}=1.0, p_{2}=2.5, \beta=0.01$, and $d=1.0$. These parameters are same to those in our previous work which simulate secondary instabilities on MHD with uniform grid system[63].

Periodic boundary condition is imposed on $x= \pm \pi$. Outflow condition $\left(\frac{\partial}{\partial y}=0\right)$ is imposed on $y= \pm 2 \pi$ or $4 \pi$, which means the y-boundary is far enough from the gradient region.

\subsection{The AMR module PAVANE}

PAVANE uses the block-based AMR. In the block-based AMR, the simulation box is decomposed into the blocks that have certain number of grid points (fig. 2). Each block is surrounded by overlapping grids which have certain width of the grid points. Calculation for the finite difference on the 
boundary region of the blocks are performed by communicating the data on the overlapping grids with the adjacent blocks. This is the same process as that of the conventional method of domain decomposition. However, in the AMR simulation, adjacent blocks can have different resolution. In the case that the resolution of the adjacent blocks is different, the data on the overlapping grids of the blocks having different resolution is interpolated. All users have to do is copy the field data, such as $\rho, \boldsymbol{v}, p$, and $\boldsymbol{B}$ in the MHD simulation, to the data structure of the PAVANE. In each block, the MHD solver runs independently. The communication process of the overlapping grids is performed by PAVANE automatically. Simultaneously, the AMR simulation is parallelized by PAVANE.

The blocks having fine structures of the field have to be refined. The condition of refinement depends on the problem that the user want to solve. Users can set an arbitrary condition of refinement on the PAVANE. Under a condition of refinement, new self-similar blocks are created inside the blocks flagged to be refined. We call the blocks refined as "parents," and the blocks created inside the parents as "children" (see figure 2 for these blocks). The number of the children in each parent is 4 (in two-dimensional system) or 8 (in three-dimensional system). Parents and children have the same number of grid points, while the width of the grid is half the size in the children. In PAVANE, the blocks are managed in a fully threaded tree (FTT) data structure [4]. The package has been applied into simple advection simulation [64].

The AMR blocks have the unique order numbers which are indicated by 64 bit integer variables. Since 6 bit out of 64 bit integer is used to memorize 


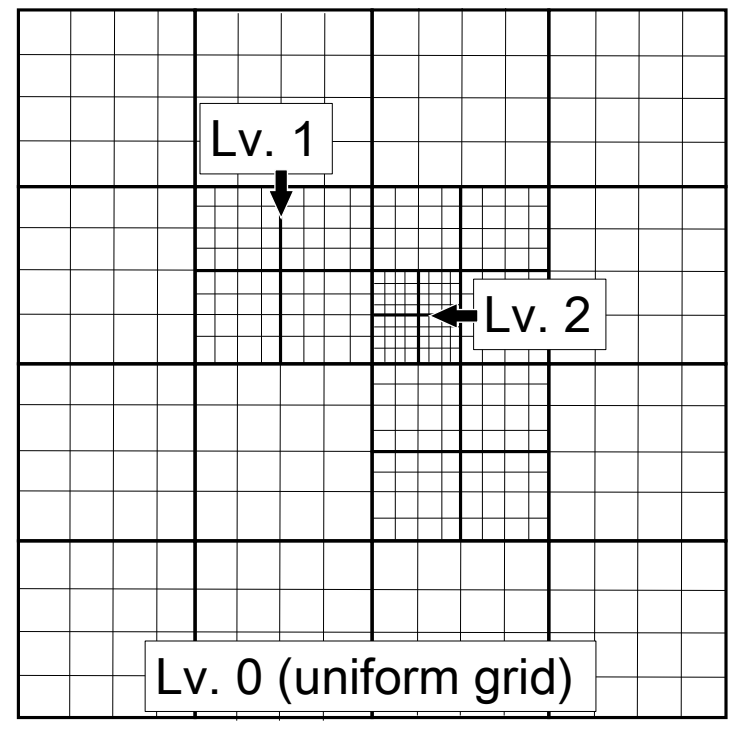

Figure 2: An example of block configuration. A block has $4 \times 4$ grid points in this case. The width of the grid becomes half as the level of AMR increase by one.

the level of AMR blocks, the upper limit of the level in the PAVANE is $2^{6}-1=63$ level. Since 57 bit out of 64 bit integer is used to memorize Morton number composed of AMR block coordinate. The unit of the AMR block coordinate is defined as the number of blocks from left side in each layers. Each dimension uses 19 bit, and then the upper limit of the number of AMR blocks per dimension is $2^{19}=524288$ blocks. The remaining 1 bit is used as the flag of error. Independently of the limit coming from the unique order numbers, the AMR block have arbitrary number of grids. However, the number of grids in the AMR block must be even number and all AMR blocks have same number of grids. Namely, self-similarity block structure is adopted. The number of grid in the $x$-direction does not have to be same as the number of the grid in the $y$-direction. In addition, the condition to 
generate and to erase higher level blocks can be freely decided by users of PAVANE module. When the AMR brocks are generated or erased, the AMR blocks are re-distributed into the multi-processes according to the unique order number of the AMR blocks. The benchmark of this load distribution is described in subsection 4.3.

\subsection{Estimation of Fourier Coefficients}

The n-th coefficient $c_{n}$ of the complex Fourier series of function $f(x)$ in the case of uniform grid system is

$$
c_{n}=\int_{-\pi}^{\pi} f(x) \exp (-i \pi x) \mathrm{d} x .
$$

To evaluate the linear growth rates in the case of AMR simulation, the integral can be split by blocks.

$$
c_{n}=\sum_{m} \int_{a(m)}^{b(m)} f(x) \exp (-i \pi x) \mathrm{d} x
$$

where $m$ is the index of the block which has the interval $[a(m), b(m)]$. We estimate the Fourier coefficients by replacing the integral in eq.(11) by numerical integration.

\section{Level-by-level Artificial Viscosity Method}

The MHD equations (eq(1)-(4)) except the artificial viscosity terms are called ideal MHD equations. They do not have any physical dissipative terms such as resistivity and heat conduction. Without dissipative terms, RTI is unstable for any wavenumber on the condition above. Namely, the numer-

ical simulation of RTI on the ideal MHD requires infinitely high resolution. 
Artificial viscosity can damp the waves having short wavelength, so that the simulation can be carried out on the finite resolution. This is a trade off between the accuracy of the simulation and the resolution of the grid. Simulations with large artificial viscosity lose reliability because they neglect short-wavelength phenomena. High accuracy simulations require both high resolution and small artificial viscosity in order not to neglect shortwavelength phenomena.

In general, artificial viscosity is used in fluid simulations to restrict the generation of the waves having short wavelength. The suitable value of the artificial viscosity under a certain condition depends on the resolution of the grid of field data. In simulations with the AMR method, the resolution of the grid of field data is determined level by level. Namely, the width of the grid of field data becomes half when the level of hierarchy of the block raises one level. If uniform artificial viscosity is used in the whole blocks in the simulation with the AMR method, the numerical values of the field data in the low-resolution blocks often diverge, while the small scale structures in the high resolution blocks are damped. Thus, it is not appropriate to employ uniform artificial viscosity on the all the blocks in the simulation with the AMR method. Therefore, the artificial viscosity should be also changed according to the level of the hierarchy of the blocks. We propose a new method to determine suitable values of artificial viscosity level by level as follows.

The level-by-level artificial viscosity $\mu$ is defined as

$$
\mu=\mu_{0}\left(\frac{1}{N}\right)^{l}
$$

where $\mu_{0}$ is the artificial viscosity in the level 0 and $l$ is the hierarchy level 
of the blocks in the simulation of the AMR. The parameter $N$ is determined by the following von Neumann stability analysis.

We consider the influence of the artificial viscosity on the numerical stability on the simulations with eq. (1)-(4). To determine the parameter $N$, we consider here a simple diffusion equation below.

$$
\frac{\partial u}{\partial t}=\mu \frac{\partial^{4} u}{\partial x^{4}}
$$

When the eq. (13) is discretized with 1st order forward difference in time and 4th order central-finite difference in space, it is rewritten by

$$
\frac{u_{j}^{n+1}-u_{j}^{n}}{\Delta t}=\mu \frac{u_{j+2}^{n}-4 u_{j+1}^{n}+6 u_{j}^{n}-4 u_{j-1}^{n}+u_{j+2}^{n}}{\Delta x^{4}}
$$

where $u_{j}^{n}$ is the discretized solution at the $\mathrm{j}$-th grid point on $\mathrm{n}$-th step in time evolution. The solution is assumed as the wave having the amplitude $a$ and the wavenumber $k$ :

$$
u_{j}^{n}=a^{n} \exp (i j k \Delta x)
$$

Substituting eq. (15) for eq. (14) results

$$
a=1-16 \mu \frac{\Delta t}{\Delta x^{4}} \sin ^{4}\left(\frac{k \Delta x}{2}\right)
$$

The stability condition become

$$
|a| \leq 1 \rightarrow \frac{\Delta t}{\Delta x^{4}} \leq \frac{1}{8 \mu}
$$

Assume that $\Delta t$ is constant, increasing the level of hierarchy of the block by one makes $\Delta x 1 / 2$, so that $\mu$ should be smaller than $1 / 16$ as the level of 
hierarchy of the block increase. Therefore, $\mathrm{N}$ of the eq. (12) should be 16, and the level-by-level artificial viscosity is the form as follows.

$$
\mu=\mu_{0}\left(\frac{1}{16}\right)^{l}
$$

We note that this conclusion does not depend on the discretization method used for the eq. (14). $\Delta x^{4}$ in the stability condition (eq. (17)) comes from the 4 th order differential in the eq. 13.

\section{Simulation Results and Discussion}

\subsection{Linear growth rate}

To confirm the validity of the level-by-level artificial viscosity method, the linear growth rates of RTI were compared between the AMR simulation and the uniform grid simulation. Of course, the AMR simulation was performed with the level-by-level artificial viscosity method, and the uniform grid simulation was performed with constant artificial viscosity. These simulation were carried out under the initial condition of MHD described in subsection 2.2 .

First, the linear growth rates of RTI were measured in the uniform grid simulation. the resolution of the uniform grid is $1024 \times 2048$. The linear growth rates were evaluated by fitting the Fourier spectra for each wavenumber. Figure 3 shows the linear growth rates measured in the uniform grid simulation for the cases that constant artificial viscosity is varied from $10^{-11}$ to $10^{-7}$. From this figure, the linear growth rates strongly depend on the artificial viscosity. In the case that the artificial viscosity is large, the linear

growth rates in high wavenumber region were remarkably reduced, while in 


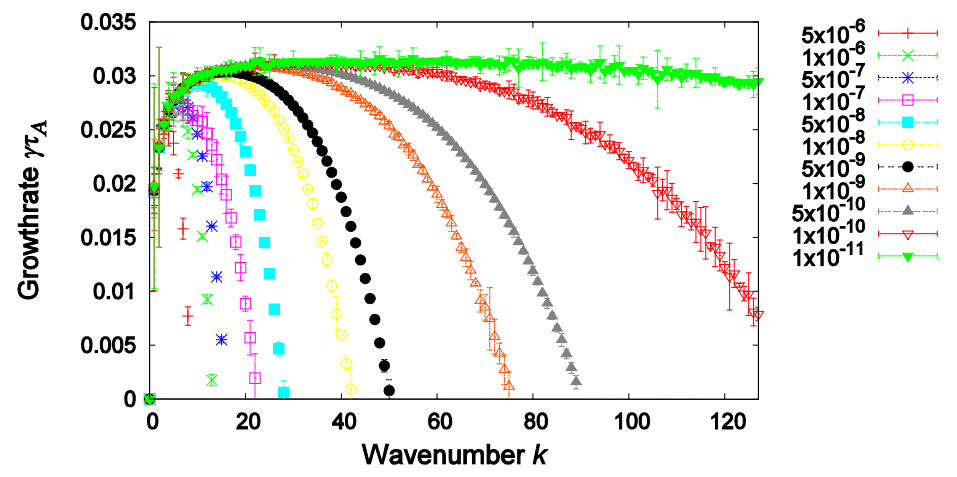

Figure 3: The growth rates, $\gamma \tau_{A}$, as functions of the wave number in the uniform grid simulations. The error bars show the square root of the variance from estimation of the growth rates.

the case of small viscosity, the linear growth rates in high wavenumber region were hardly reduced.

The resolution of the uniform grid simulation is enough to represent fine structures because actually our previous work observed secondary instabilities in the same resolution. However, fig. 3 implies that to represent fine structures, not only the grid of high resolution and also suitable artificial viscosity.

Although the suitable value of artificial viscosity depends on the resolution of grids, the AMR blocks in the different resolution have different resolution. Then, the artificial viscosity of each AMR blocks is set by the level-by-level artificial viscosity method. If the level-by-level artificial viscosity is valid, the linear growth rates measured in AMR simulation become comparable to the linear growth rates measured in the uniform grid simulation. 
The linear growth rates measured in the AMR simulation with the levelby-level artificial viscosity are shown in fig. 4. The maximum level of AMR is restricted up to 4 , which means there are 5 levels of hierarchy in the grid. Each block has $32 \times 32$ grid points, and is surrounded by overlapping grid whose width is 4 grid points. The resoluation in the level zero layer is $256 \times$ 512. The condition of refinement is whether the density difference between adjacent grids is larger than $10^{-4}$ or not. When the AMR block in the level two layer is generated, the resolution is corresponding to $1024 \times 2048$, which is same as the resolution in the above uniform grid simulation. The artificial viscosity of level zero block $\mu_{0}=1.0 \times 10^{-7}$. To clarify the effect of the levelby-level artificial viscosity, we calculated the four cases that the maximum level of the AMR blocks is restricted up to 1,2,3, and 4. Here, the five solid lines shown in fig 4 indicate the linear growth rates measured in the uniform grid simulations in the cases that the constant artificial viscosity is set to $\mu_{0}, \mu_{0} / 16, \mu_{0} / 16^{2}, \mu_{0} / 16^{3}$, and $\mu_{0} / 16^{4}$ which are corresponding to the value of the level-by-level artificial viscosity in the zeroth to fourth AMR layer. It is clearly confirmed that the linear growth rates measured in the AMR simulations are good agreement with the linear growth rates measured in the uniform grid simulation in the corresponding constant artificial viscosity. This result suggest that the level-by-level artificial viscosity method enables to represent linear growth rates comparable to uniform grid simulations with high resolution and enough smaller artificial viscosity.

\subsection{Non-linear growth stage}

Furthermore, the generation of fine structures in the nonlinear growth stage is confirmed in AMR simulation with level-by-level artificial viscosity. 


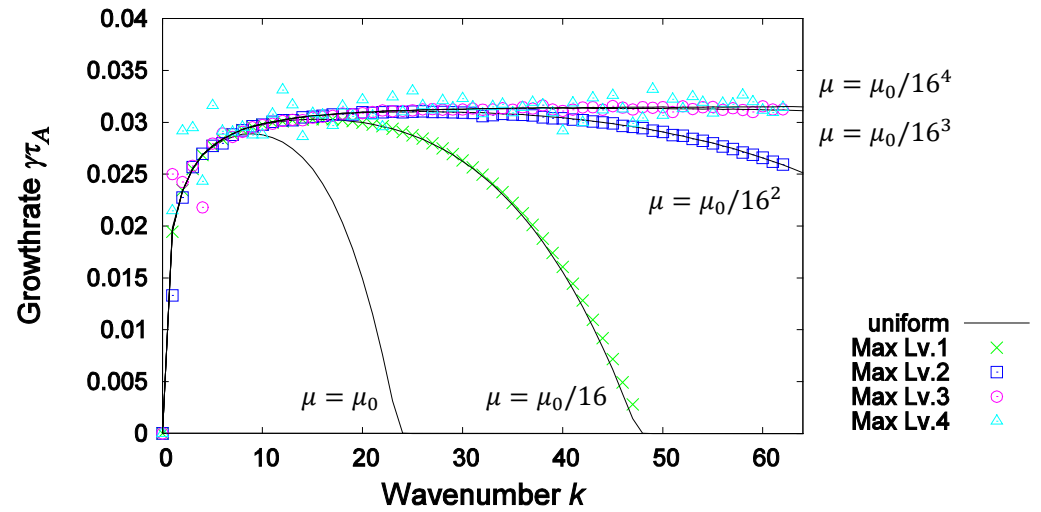

Figure 4: The growth rates, $\gamma \tau_{A}$, as functions of the wave number in the AMR simulations with the level-by-level artificial viscosity (colored points). The difference of colors shows the difference of the maximum level of AMR. The black solid lines show the growth rates measured in the uniform grid simulations with constant artificial viscosities $\mu_{0}, \mu_{0} / 16$, $\mu_{0} / 16^{2}, \mu_{0} / 16^{3}$, and $\mu_{0} / 16^{4}$. 
The simulation condition is same as the AMR simulation for linear growth rates analysis in subsection 4.1. However, the resoluation in the level zero layer is $256 \times 1024$. When the AMR block in the level four layer is generated, the resolution is corresponding to $4096 \times 16384$.

Fig. 5 shows density profiles of nonlinear growth stage of RTI simulations in AMR simulation. The three kind of AMR simulation in the cases that the parameter $N$ is set to $4((\mathrm{a})$ in fig. 5), 10((b) in fig. 5), and $16((\mathrm{c})$ in fig. 5) were compared. From the von Neumann stability analysis in previous section, the suitable parameter for $N$ is 16 . As $N$ increase, finer structure of secondary instability appears at the surface of the primary instability. It is confirmed that the artificial viscosities in the cases that $\mathrm{N}=4$ and 10 are too large to represent fine structures of secondary instabilities. On the other hand, in the case that $N=16$, the fine structure of the secondary instabilities of both the secondary instability of RTI and Kelvin-Helmholtz(KH) like structures were observed place by place as shown in fig. 6. The AMR simulation in the case that $N=25$ was also calculated, and then field data calculated in the simulation diverged numerically. Consequently, the artificial viscosity in the case that $N=25$ is too small to damp the waves having high wavenumber. These results suggest that choice of $N=16$ according to the von Neumann stability analysis is valid. Therefore, the method of level-by-level artificial viscosity also works well on the AMR simulation for the nonlinear growth stage.

\subsection{Benchmark of load distribution}

Here, we performed the benchmark of load distribution of the AMR simulation on the multi-processers. The target AMR simulation is the present 

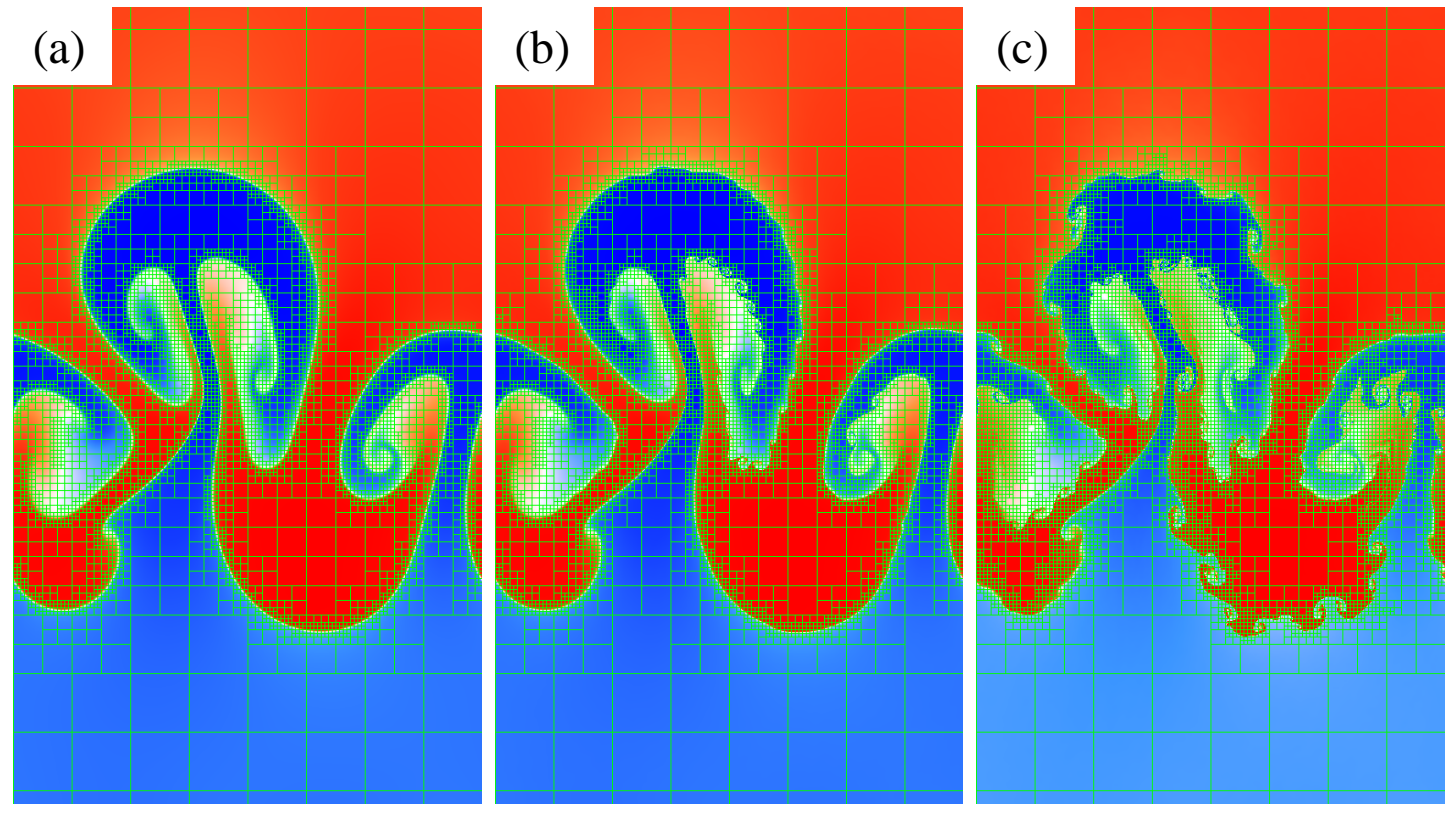

Figure 5: Density profiles of nonlinear growth stage of RTI simulations with AMR and level-by-level artificial viscosity (eq. (12)) of $\mathrm{N}=4(\mathrm{a}), 10(\mathrm{~b})$, and 16(c) with maximum level 4. These figure are snap shots at $240.0 \tau_{A}$. 


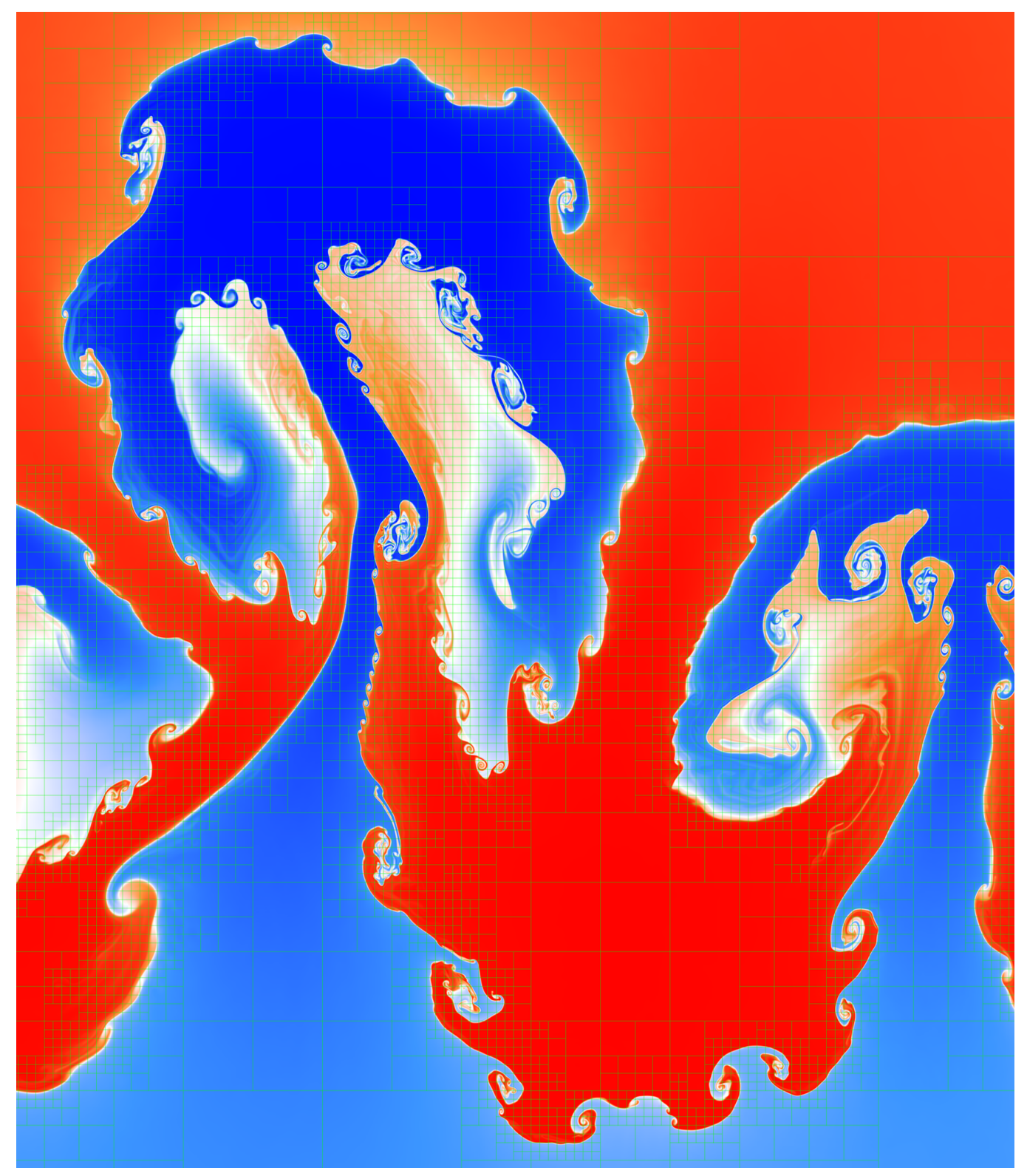

Figure 6: The enlarged view of snapshot of the AMR simulation shown in Fig. 5(c). 
RTI of MHD system for the non-linear growth stage described in subsection 4.2. Initially, the level zero blocks are distributed into all processes evenly. In this benchmark, we compared the following two conditions. In the first condition, although the level zero block is initially distributed into all processes, newly generated high level blocks are calculated by the process of their parent processes, which is labeled gwithout load balanceh in the fig. 7 . In the second condition, all blocks are re-distributed into all processes evenly whenever a new block is generated. It is confirmed that the second condition with load balance is about five times faster than the first condition without load balance. Figure 7(a) shows the calculation time per step as a function of elapsed time. The calculation time increases with the elapsed time because the number of AMR blocks also increases with the elapsed times as shown in fig. 7(b). From the fig. 7(a), it is confirmed that the second condition with load balance is about five times faster than the first condition without load balance. Especially important, in the second condition with load balance, the calculation time per block remains substantially constant as shown in fig. 7(c), while in the first condition without load balance, the calculation time per block increases. This is caused by the following reason. The calculation time includes the waiting time to wait calculation in another processes. In the first condition without load balance, the waiting time in the processes which have few AMR blocks become long. As a result, the calculation time per block increases. On the other hand, in the condition with load balance, the waiting time becomes small, and then the calculation time per block can keep a constant value.

In the present application into two dimensional MHD system, the calcu- 
lation cost of grid quantities in blocks is not too greater than the communications cost for margin region between processes. The ratio of the communication cost to the calculation cost becomes larger in the three dimensional system. Moreover, the number of total blocks in the two dimensional system is much smaller than that of the three dimensional system. For all that, our AMR module PAVANE has achieved good load distribution. Therefore, we expect good performance in also the three dimensional system. Of course, our AMR library can treat much greater number of blocks, we will perform not only the benchmark of load distribution but also the benchmarks of strong and week scaling in future works.

\section{Visualization and analysis}

Visualization and analysis of hierarchical data calculated from simulations with the AMR method are more difficult than those calculated from simulations with uniform grid. To support users to analyze the AMR data, PAVANE has two types of visualization and analysis tool. One is in-situ image output function and the other is a visualization software dedicated to the package.

The in-situ image output function can be called in the code, and make image files at run time. Users have only to choose the color of the image and the resolution of the image. Actually, fig. 5 were made by the in-situ image output function.

To analyze the data in detail, the package provide a visualization software named "Unique Interface for HierArchical Numerical data (UIHAN)". This software visualizes the block data calculated from the simulation with the 
(a)

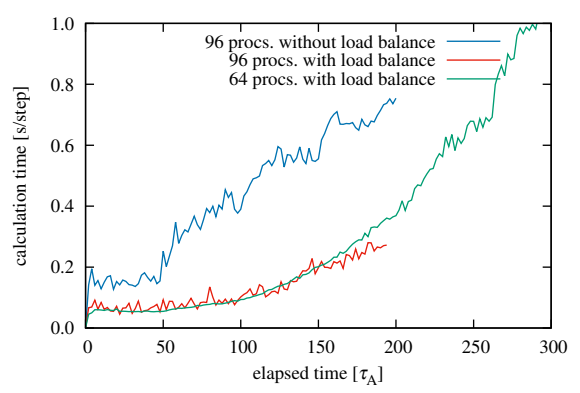

(c)

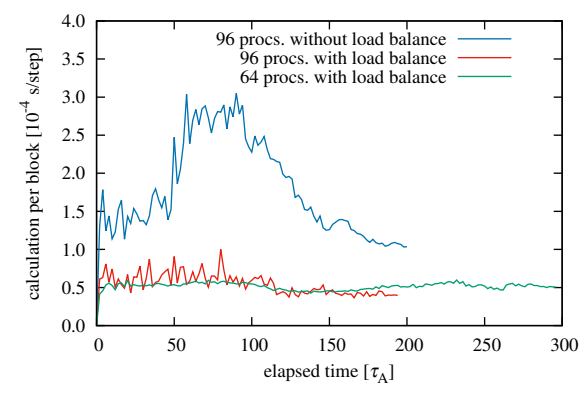

(b)

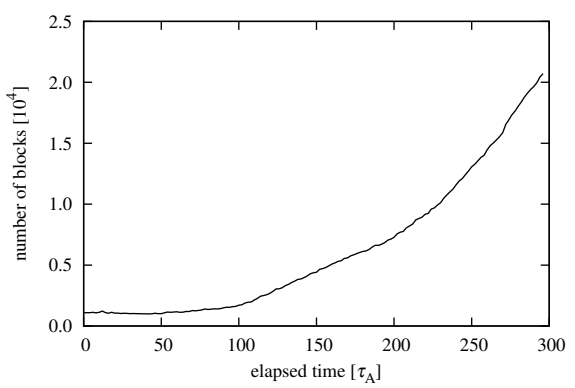

Figure 7: (a) The calculation time, (b) the total number of AMR blocks, and (c) the calculation time per AMR blocks as functions of elapsed time. The target application is the AMR simulation for the non-linear growth stage shown in fig. 5(c) and fig. 6. The target computer systems are the system of 64 processes which is composed of 16 processes $\times 4$ nodes and the system of 96 processes which is composed of 24 processes $\times 4$ nodes. 
AMR method. The visualized block data is floating in three-dimensional space. The difference of the level of AMR blocks is visualized as layered surfaces (see fig. 8(a)). The viewpoint can be moved freely. The blocks can be magnified to draw fine structures on the high-resolution grid (fig. 8(b)). By operating the left slider, the plot-over-line moves on the simulation box. The field data and its Fourier spectrum along with the plot-over-line are drawn in the left side of the screen. The direction of the plot-over-line can be changed $\mathrm{x}$-direction to $\mathrm{y}$-direction. These functions make it possible to analyze the data quantitatively, even if the block data structures in AMR simulation have different resolutions.

\section{Summary}

AMR method is a powerful tool for allocating high resolution grids only to the region having complicated field structure within limited computational resources. We are developing the package for the AMR method, PAVANE. PAVANE is applied to the MHD simulation code. For the treatment of artificial viscosity in the simulations with the AMR method, we propose

the new technique, "level-by-level artificial viscosity" method. Numerical simulation of RTI on MHD is carried out using the AMR method and levelby-level artificial viscosity method. It is confirmed that level-by-level artificial viscosity method works well on both linear growth stage and nonlinear growth stage of RTI in the AMR simulation.

We have limited ourselves to two-dimensional simulations in this paper. We are considering a three-dimensional version as future work. 


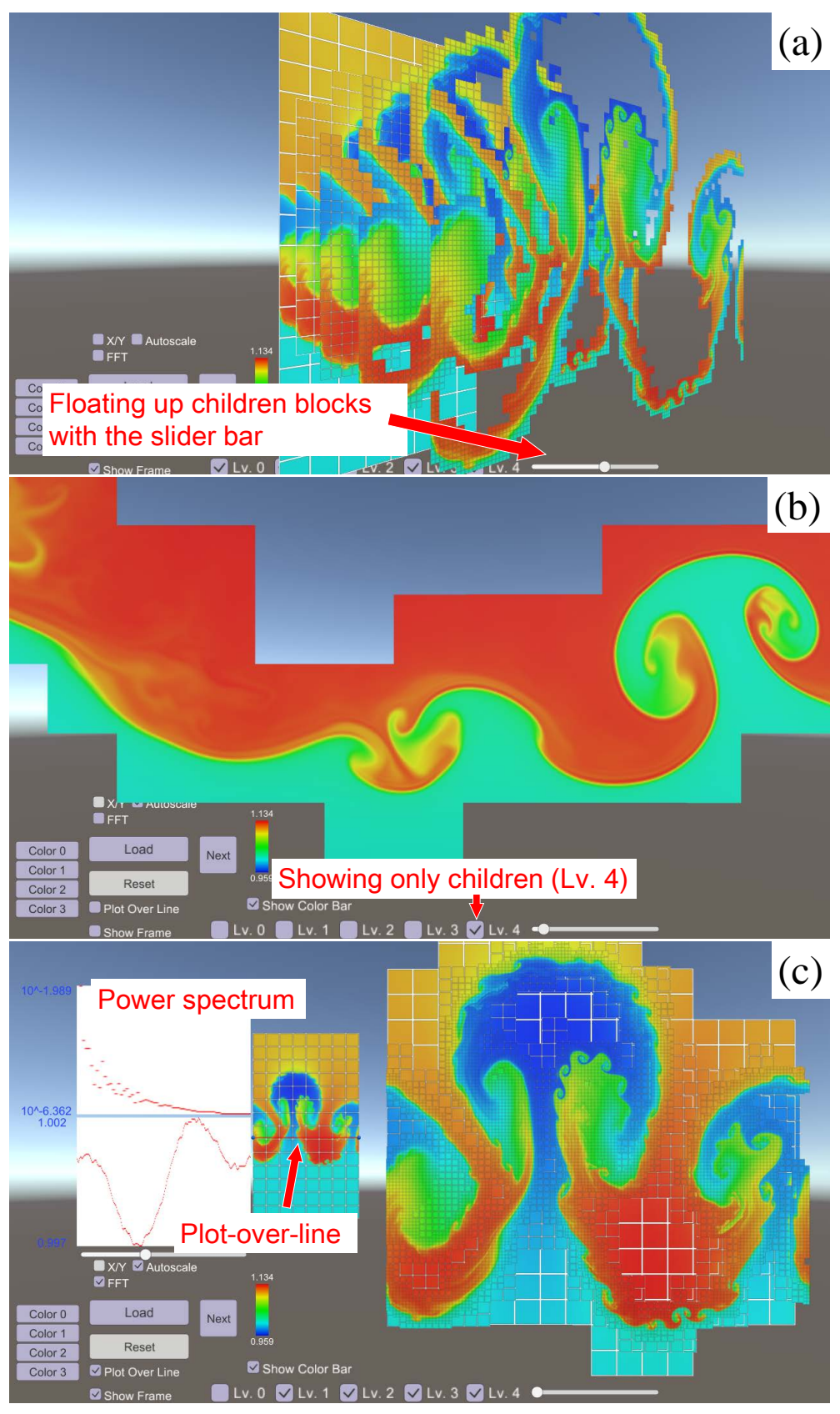

Figure 8: (a) shows the children blocks floating in 3D space. (b) shows children blocks magnified. (c) shows the graphs plotted with the plot-over-line function and FTT function. 


\section{Acknowledgements}

Numerical simulations in this work were carried out on the Plasma Simulator at National Institute for Fusion Science (NIFS) and the Helios supercomputer system at Computational Simulation Centre of International Fusion Energy Research Centre (IFERC-CSC). This work was partially supported by KAKENHI(26820398, 15H05563), NIFS Collaboration Research

program (NIFS14KNST061), and the Japan Science and Technology Agency, Core Research for Evolutional Science and Technology (JST/CREST).

\section{References}

[1] M. J. Berger, J. Oliger, Adaptive mesh refinement for hyperbolic partial differential equations, J. Comput. Phys. 53 (3) (1984) 484-512. doi:10.1016/0021-9991(84)90073-1.

[2] D. De Zeeuw, K. G. Powell, An adaptively-refined Cartesian mesh solver for the Euler equations (1991). doi:10.1006/jcph.1993.1007.

[3] W. J. Coirier, K. G. Powell, Solution-adaptive Cartesian cell approach for viscous and inviscid flows, AIAA J. 34 (5) (1996) 938-945. doi:10.2514/3.13171.

[4] A. M. Khokhlov, Fully Threaded Tree for Adaptive Refinement Fluid Dynamics Simulations 543 (1997) 22. arXiv:9701194, doi:10.1006/jcph.1998.9998.

[5] A. L. Garcia, J. B. Bell, W. Y. Crutchfield, B. J. Alder, Adaptive Mesh 
and Algorithm Refinement Using Direct Simulation Monte Carlo, J. Comput. Phys. 154 (1) (1999) 134-155. doi:10.1006/jcph.1999.6305.

[6] H. T. Ahn, M. Shashkov, Adaptive moment-of-fluid method, J. Comput. Phys. 228 (8) (2009) 2792-2821. doi:10.1016/j.jcp.2008.12.031.

[7] D. Fuster, G. Agbaglah, C. Josserand, S. Popinet, S. Zaleski, Numerical simulation of droplets, bubbles and waves: state of the art, Fluid Dyn. Res. 41 (6) (2009) 065001. doi:10.1088/0169-5983/41/6/065001.

[8] H. Ji, F. Lien, E. Yee, A new adaptive mesh refinement data structure with an application to detonation, J. Comput. Phys. 229 (23) (2010) 8981-8993. doi:10.1016/j.jcp.2010.08.023.

[9] S. Zabelok, R. Arslanbekov, V. Kolobov, Adaptive Kinetic-Fluid Solvers for Heterogeneous Computing Architectures arXiv:1503.00707.

[10] K. G. Powell, P. L. Roe, T. J. Linde, T. I. Gombosi, D. L. De Zeeuw, A Solution-Adaptive Upwind Scheme for Ideal Magnetohydrodynamics, J. Comput. Phys. 154 (2) (1999) 284-309. doi:10.1006/jcph.1999.6299.

[11] D. L. De Zeeuw, T. I. Gombosi, C. P. T. Groth, K. G. Powell, Q. F. Stout, An adaptive MHD method for global space weather simulations, IEEE Trans. Plasma Sci. 28 (6) (2000) 1956-1965. doi:10.1109/27.902224.

[12] T. Gombosi, D. DeZeeuw, C. Groth, K. Powell, Magnetospheric configuration for Parker-spiral IMF conditions: Results of a 3D AMR MHD simulation, Adv. Sp. Res. 26 (1) (2000) 139-149. doi:10.1016/S02731177(99)01040-6. 
[13] D. S. Balsara, C. D. Norton, Highly parallel structured adaptive mesh refinement using parallel language-based approaches, Parallel Comput. 27 (1-2) (2001) 37-70. doi:10.1016/S0167-8191(00)00088-0.

[14] D. Balsara, Second Order Accurate Schemes for Magnetohydrodynamics With Divergence-Free Reconstruction (2003) 85arXiv:0308249, doi:10.1086/381377.

[15] J. Kleimann, A. Kopp, H. Fichtner, R. Grauer, K. Germaschewski, Three-dimensional MHD high-resolution computations with CWENO employing adaptive mesh refinement, Comput. Phys. Commun. 158 (1) (2004) 47-56. doi:10.1016/j.comphy.2003.12.003.

[16] D. Rosenberg, A. Fournier, P. Fischer, A. Pouquet, Geophysicalastrophysical spectral-element adaptive refinement (GASpAR): Objectoriented h-adaptive fluid dynamics simulation, J. Comput. Phys. 215 (1) (2006) 59-80. arXiv:0507402, doi:10.1016/j.jcp.2005.10.031.

[17] D. S. Balsara, Divergence-Free Reconstruction of Magnetic Fields and WENO Schemes for Magnetohydrodynamics arXiv:0811.2192.

[18] U. Ziegler, The NIRVANA code: Parallel computational MHD with adaptive mesh refinement, Comput. Phys. Commun. 179 (4) (2008) 227244. doi:10.1016/j.cpc.2008.02.017.

[19] C. Jiang, X. Feng, J. Zhang, D. Zhong, AMR Simulations of Magnetohydrodynamic Problems by the CESE Method in Curvilinear Coordinates, Sol. Phys. 267 (2) (2010) 463-491. doi:10.1007/s11207-010-9649-6. 
[20] A. V. Kravtsov, A. A. Klypin, A. M. Khokhlov, Adaptive Refinement Tree - a new high-resolution N-body code for cosmological simulations (1997) 1-41arXiv:9701195, doi:10.1086/313015.

[21] T. Abel, G. L. Bryan, M. L. Norman, The Formation and Fragmentation of Primordial Molecular Clouds 1 (1998) 5. arXiv:9810215, doi:10.1086/309295.

[22] M. L. Norman, G. L. Bryan, Cosmological Adaptive Mesh Refinement, Numer. Astrophys. Proc. Int. Conf. Numer. Astrophys. 1998240 (1998) 14. arXiv:9807121.

[23] J. K. Truelove, R. I. Klein, C. F. McKee, J. H. Holliman II, L. H. Howell, J. A. Greenough, D. T. Woods, Self-gravitational Hydrodynamics with Three-dimensional Adaptive Mesh Refinement: Methodology and Applications to Molecular Cloud Collapse and Fragmentation, Astrophys. J. 495 (2) (1998) 821-852. doi:10.1086/305329.

[24] R. Teyssier, Cosmological hydrodynamics with adaptive mesh refinement, Astron. Astrophys. 385 (1) (2002) 337-364. arXiv:0111367, doi:10.1051/0004-6361:20011817.

[25] F. Miniati, D. F. Martin, Constrained-Transport Magnetohydrodynamics With Adaptive Mesh Refinement in Charm, Astrophys. J. Suppl. Ser. 195 (1) (2011) 5. arXiv:arXiv:1103.1878v1, doi:10.1088/0067$0049 / 195 / 1 / 5$.

[26] K. Clough, P. Figueras, H. Finkel, M. Kunesch, E. A. Lim, S. Tunya- 
suvunakool, GRChombo : Numerical Relativity with Adaptive Mesh Refinement arXiv:1503.03436.

[27] P. Song, D. L. DeZeeuw, T. I. Gombosi, C. P. T. Groth, K. G. Powell, A numerical study of solar wind-magnetosphere interaction for northward interplanetary magnetic field, J. Geophys. Res. 104 (A12) (1999) 28361. doi:10.1029/1999JA900378.

[28] M. Anderson, E. Hirschmann, S. L. Liebling, D. Neilsen, Relativistic MHD with Adaptive Mesh Refinement (2006) 24arXiv:0605102, doi:10.1088/0264-9381/23/22/025.

[29] S. Fromang, P. Hennebelle, R. Teyssier, A High Order Godunov Scheme with Constrained Transport and Adaptive Mesh Refinement for Astrophysical MHD 384 (2006) 17. arXiv:0607230, doi:10.1051/00046361:20065371.

[30] T. Matsumoto, Self-gravitational Magnetohydrodynamics with Adaptive Mesh Refinement for Protostellar Collapse (2002) (2006) 23. arXiv:0609105.

[31] A. J. Cunningham, A. Frank, P. Varniere, S. Mitran, T. W. Jones, Simulating Magnetohydrodynamical Flow with Constrained Transport and Adaptive Mesh Refinement; Algorithms \& Tests of the AstroBEAR Code (2007) 519-542arXiv:0710.0424, doi:10.1088/0067$0049 / 182 / 2 / 519$.

[32] F. Miniati, P. Colella, Block structured adaptive mesh and time re- 
finement for hybrid, hyperbolic + N-body systems, J. Comput. Phys. 227 (1) (2007) 400-430. arXiv:0608156, doi:10.1016/j.jcp.2007.07.035.

[33] B. van der Holst, R. Keppens, Hybrid block-AMR in cartesian and curvilinear coordinates: MHD applications, J. Comput. Phys. 226 (1) (2007) 925-946. doi:10.1016/j.jcp.2007.05.007.

[34] C. Zanni, A. Ferrari, R. Rosner, G. Bodo, S. Massaglia, MHD simulations of jet acceleration from Keplerian accretion disks: the effects of disk resistivity 828 (2007) 20. arXiv:0703064, doi:10.1051/00046361:20066400.

[35] D. F. Duffin, R. E. Pudritz, Simulating hydromagnetic processes in star formation: Introducing ambipolar diffusion into an adaptive mesh refinement code, Mon. Not. R. Astron. Soc. 391 (4) (2008) 1659-1673. arXiv:0810.0299, doi:10.1111/j.1365-2966.2008.14026.x.

[36] P. Hennebelle, A. Ciardi, Disk formation during collapse of magnetized protostellar cores 32 (2009) 29-33. arXiv:0909.3190, doi:10.1051/0004$6361 / 200913008$.

[37] B. Commercon, P. Hennebelle, E. Audit, G. Chabrier, R. Teyssier, Radiative, magnetic and numerical feedbacks on small-scale fragmentation 3 (2010) 4. arXiv:1011.5651, doi:10.1051/0004-6361/200913597.

[38] Z. B. Etienne, Y. T. Liu, S. L. Shapiro, Relativistic magnetohydrodynamics in dynamical spacetimes: A new adaptive mesh refinement implementation, Phys. Rev. D - Part. Fields, Gravit. Cosmol. 82 (8) (2010) 1-21. arXiv:1007.2848, doi:10.1103/PhysRevD.82.084031. 
[39] A. Mignone, C. Zanni, P. Tzeferacos, B. van Straalen, P. Colella, G. Bodo, The Pluto Code for Adaptive Mesh Computations in Astrophysical Fluid Dynamics, Astrophys. J. Suppl. Ser. 198 (1) (2011) 7. arXiv:1110.0740, doi:10.1088/0067-0049/198/1/7.

[40] R. L. Jiang, C. Fang, P. F. Chen, A new MHD code with adaptive mesh refinement and parallelization for astrophysics, Comput. Phys. Commun. 183 (8) (2012) 1617-1633. arXiv:1204.5849, doi:10.1016/j.cpc.2012.02.030.

[41] J. Müller, S. Simon, Y. C. Wang, U. Motschmann, D. Heyner, J. Schüle, W. H. Ip, G. Kleindienst, G. J. Pringle, Origin of Mercury's double magnetopause: 3D hybrid simulation study with A.I.K.E.F., Icarus 218 (1) (2012) 666-687. doi:10.1016/j.icarus.2011.12.028.

[42] M. Matsumoto, H. Usui, M. Nunami, M. Nakamura, I. Shinohara, Two-Dimensional AMR-PIC Plasma Simulation for MiniMagnetosphere of Magnetized Object, Plasma Fusion Res. 8 (2013) 2406132. doi:10.1585/pfr.8.2406132.

[43] O. Zanotti, M. Dumbser, A high order special relativistic hydrodynamic code with space-time adaptive mesh refinement, Comput. Phys. Commun. 188 (2013) 21. arXiv:1312.7784, doi:10.1016/j.cpc.2014.11.015.

[44] G. L. Bryan, M. L. Norman, B. W. O'Shea, T. Abel, J. H. Wise, M. J. Turk, D. R. Reynolds, D. C. Collins, P. Wang, S. W. Skillman, B. Smith, R. P. Harkness, J. Bordner, J.-h. Kim, M. Kuhlen, H. Xu, N. Goldbaum, C. Hummels, A. G. Kritsuk, E. Tasker, S. Skory, C. M. Simpson, 
O. Hahn, J. S. Oishi, G. C. So, F. Zhao, R. Cen, Y. Li, Enzo: an Adaptive Mesh Refinement Code for Astrophysics, Astrophys. J. Suppl. Ser. 211 (2) (2014) 19. arXiv:1307.2265, doi:10.1088/0067-0049/211/2/19.

[45] X. Feng, C. Xiang, D. Zhong, Y. Zhou, L. Yang, X. Ma, SIP-CESE MHD model of solar wind with adaptive mesh refinement of hexahedral meshes, Comput. Phys. Commun. 185 (7) (2014) 1965-1980. doi:10.1016/j.cpc.2014.03.027.

[46] J. Wang, X. Feng, A. Du, Y. Ge, Modeling the interaction between the solar wind and Saturn's magnetosphere by the AMR-CESEMHD method, J. Geophys. Res. Sp. Phys. 119 (12) (2014) 9919-9930. doi:10.1002/2014JA020420.

[47] C. Jablonowski, R. C. Oehmke, Q. F. Stout, Block-structured adaptive meshes and reduced grids for atmospheric general circulation models, Philos. Trans. A. Math. Phys. Eng. Sci. 367 (1907) (2009) 4497-4522. doi:10.1098/rsta.2009.0150.

[48] E. Blayo, L. Debreu, Adaptive Mesh Refinement for Finite-Difference Ocean Models: First Experiments, J. Phys. Oceanogr. 29 (6) (1999) 1239-1250. doi:10.1175/1520-0485(1999)029;1239:AMRFFD ¿2.0.CO;2.

[49] E. Santilli, A. Scotti, The Stratified Ocean Model with Adaptive Refinement, J. Comput. Phys. 291 (2015) 60-81. doi:10.1016/j.jcp.2015.03.008.

[50] Y. L. Y. Liu, C. Sarris, Fast time-domain simulation of optical waveguide structures with a multilevel dynamically adaptive mesh 
refinement FDTD approach, J. Light. Technol. 24 (2006) 1-13. doi:10.1109/JLT.2006.876899.

[51] V. Sizyuk, A. Hassanein, Integrated self-consistent analysis of NSTX performance during normal operation and disruptions, J. Nucl. Mater. 438 (SUPPL) (2013) S809--S813. doi:10.1016/j.jnucmat.2013.01.175.

[52] J. Zhang, M. J. Ni, A consistent and conservative scheme for MHD flows with complex boundaries on an unstructured Cartesian adaptive system, J. Comput. Phys. 256 (2014) 520-542. doi:10.1016/j.jcp.2013.08.004.

[53] [link].

URL https://ccse.lbl.gov/index.html

[54] [link].

URL http://cactuscode.org/

[55] T. Goodale, G. Allen, G. Lanfermann, J. Massó, T. Radke, E. Seidel, J. Shalf, The Cactus Framework and Toolkit: Design and Applications, in: High Perform. Comput. Comput. Sci. - VECPAR 2002, 2003, pp. 197-227. doi:10.1007/3-540-36569-9_13.

[56] [link].

URL https://commons.lbl.gov/display/chombo/Chombo+-+

Software+for+Adaptive+Solutions+of+Partial+Differential+ Equations

[57] [link].

URL http://www.physics.drexel.edu/ olson/paramesh-doc/ Users_manual/amr.html 
[58] P. MacNeice, K. M. Olson, C. Mobarry, R. De Fainchtein, C. Packer, PARAMESH: A parallel adaptive mesh refinement community toolkit, Comput. Phys. Commun. 126 (2000) 330-354. doi:10.1016/S00104655(99)00501-9.

[59] [link].

URL http://uintah.utah.edu/

[60] B. Fryxell, K. Olson, P. Ricker, F. X. Timmes, M. Zingale, D. Q. Lamb, P. MacNeice, R. Rosner, J. W. Truran, H. Tufo, FLASH: An Adaptive Mesh Hydrodynamics Code for Modeling Astrophysical Thermonuclear Flashes, Astrophys. J. Suppl. Ser. 131 (1) (2000) 273-334. doi:10.1086/317361.

[61] F. Löffler, J. Faber, E. Bentivegna, T. Bode, P. Diener, R. Haas, I. Hinder, B. C. Mundim, C. D. Ott, E. Schnetter, G. Allen, M. Campanelli, P. Laguna, The Einstein Toolkit: a community computational infrastructure for relativistic astrophysics, Class. Quantum Gravity 29 (11) (2012) 115001. arXiv:1111.3344, doi:10.1088/0264-9381/29/11/115001.

[62] R. Goto, H. Miura, A. Ito, M. Sato, T. Hatori, Hall and Gyro-Viscosity Effects on the Rayleigh-Taylor Instability in a 2D Rectangular Slab, Plasma Fusion Res. 9 (2014) 1403076.

[63] R. Goto, H. Miura, A. Ito, M. Sato, T. Hatori, Formation of large-scale structures with sharp density gradient through Rayleigh-Taylor growth in a two-dimensional slab under the two-fluid and finite Larmor radius effects, Phys. Plasmas 22 (3) (2015) 032115. doi:10.1063/1.4916061. 
[64] H. Usui, A. Nagara, M. Nunami, M. Matsumoto, Development of a Computational Framework for Block-based AMR Simulations, Procedia Comput. Sci. 29 (2014) 2351-2359. doi:10.1016/j.procs.2014.05.219. 